\title{
A Problem of a Semi-Infinite Medium Subjected to Exponential Heating Using a Dual-Phase-Lag Thermoelastic Model
}

\author{
Ahmed Elsayed Abouelregal \\ Department of Mathematics, Faculty of Science, Mansoura University, Mansoura, Egypt \\ E-mail:ahabogal@mans.edu.eg \\ Received January 2, 2011; revised March 39, 2011; accepted April 2, 2011
}

\begin{abstract}
The problem of a semi-infinite medium subjected to thermal shock on its plane boundary is solved in the context of the dual-phase-lag thermoelastic model. The expressions for temperature, displacement and stress are presented. The governing equations are expressed in Laplace transform domain and solved in that domain. The solution of the problem in the physical domain is obtained by using a numerical method for the inversion of the Laplace transforms based on Fourier series expansions. The numerical estimates of the displacement, temperature, stress and strain are obtained for a hypothetical material. The results obtained are presented graphically to show the effect phase-lag of the heat flux $\tau_{q}$ and a phase-lag of temperature gradient $\tau_{\theta}$ on displacement, temperature, stress.
\end{abstract}

Keywords: Generalized Thermoelasticity, Dual-Phase-Lag Model, Semi-Infinite Medium, Laplace Transform

\section{Introduction}

Biot [1] (1956) introduced the theory of coupled thermoelasticity to overcome the first shortcoming in the classical uncoupled theory of thermoelasticity where it predicts two phenomena not compatible with physical observations. First, the equation of heat conduction of this theory does not contain any elastic terms. Second, the heat equation is of a parabolic type, predicting infinite speeds of propagation for heat waves. The governing equations for Biot theory are coupled, eliminating the first paradox of the classical theory. However, both theories share the second shortcoming since the heat equation for the coupled theory is also parabolic.

Thermoelasticity theories that predict a finite speed for the propagation of thermal signals have aroused much interest in the last three decades. These theories are known as generalized therrnoelasticity theories. The first generalizations of the thermoelasticity theory is due to Lord and Shulman [2] who introduced the theory of generalized thermoelasticity with one relaxation time by postulating a new law of heat conduction to replace the classical Fourier' law. This law contains the heat flux vector as well as its time derivative. It contains also a new constant that acts as a relaxation time. The heat equation of this theory is of the wave-type, ensuring finite speeds of propagation for heat and elastic waves. The remaining governing equations for this theory, namely, the equations of motion and the constitutive relations remain the same as those for the coupled and the uncoupled theories. This theory was extended by Dhaliwal and Sherief [3] to general anisotropic media in the presence of heat sources.

A generalization of this inequality was proposed by Green and Laws [4] Green and Lindsay obtained another version of the constitutive equations in [5]. The theory of thermoelasticity without energy dissipation is another generalized theory and was formulated by Green and Naghdi [6]. It includes the thermal displacement gradient among its independent constitutive variables, and differs from the previous theories in that it does not accommodate dissipation of thermal energy.

Tzou $[7,8]$ proposed the dual-phase-lag (DPL) model, which describes the interactions between phonons and electrons on the microscopic level as retarding sources causing a delayed response on the macroscopic scale. For macroscopic formulation, it would be convenient to use the DPL mode for investigation of the micro-structural 
effect on the behavior of heat transfer. The physical meanings and the applicability of the DPL mode have been supported by the experimental results [9]. The dual-phase-lag (DPL) proposed by Tzou [9] is such a modification of the classical thermoelastic model in which the Fourier law is replaced by an approximation to a modified Fourier law with tow different time translations: a phase-lag of the heat flux $\tau_{q}$ and a phase-lag of temperature gradient $\tau_{\theta}$. A Taylor series approximation of the modified Fourier law, together with the remaining field equations leads to a complete system of equations describing a dual-phase-lag thermoelastic model. The model transmits thermoelastic disturbance in a wave-like manner if the approximation is linear with respect to $\tau_{q}$ and $\tau_{\theta}$, and $0 \leq \tau_{\theta}<\tau_{q}$; or quadratic in $\tau_{q}$ and linear in $\tau_{\theta}$, with $\tau_{q}>0$ and $\tau_{\theta}>0$. This theory is developed in a rational way to produce a fully consistent theory which is able to incorporate thermal pulse transmission in a very logical manner.

Danilovskaya [10] was the first to solve an actual problem in the theory of elasticity with nonuniform heat. The problem was for a half-space subjected to a thermal shock in the context of what became known as the theory of uncoupled thermoelasticity. Chandrasekharaiah and Srinath [11] studied the one dimensional thermal wave propagation in a half space based on the theory of thermoelasticity without energy dissipation due to a constant step in temperature applied to the boundary. Roychoudhuri and Dutta [12] studied thermoelastic interactions in an isotropic homogeneous thermoelastic solid containing time-dependent distributed heat sources which vary periodically for a finite time interval in the context of Green and Naghdi theory. Sherief and Dhaliwal [13] solved a generalized one-dimensional thermal-shock problem for small times. Allam et al. [14] discussed magneto-thermoelasticity for an infinite body with a spherical cavity and variable material properties without energy dissipation.

The present paper is organized as follows. Section 2 describes the formulation of the problem and the governing equations. Section 3 discusses the Laplace transform technique and the solution in the transformed domain is obtained using a potential function. Section 4 summarizes the inverse Laplace transforms using a numerical method based on Fourier expansion techniques. The last section is devoted to the numerical example for finding the temperature, displacement and the stress. These distributions are also depicted graphical.

\section{Formulation of the Problem}

We shall consider a homogeneous, isotropic, thermoelastic solid, occupying the region $x \geq 0$ where the $x$-axis is taken perpendicular to the bounding plane of the halfspace pointing inwards. The boundary conditions for temperature is in the form of exponential heating, a more realistic situation. It is assumed that the state of the medium depends only on $x$ and $t$ and that the displacement vector has components $(u(x, t), 0,0)$.

The equation of motion in the absence of body forces in the one dimensional case has the form

$$
\rho \frac{\partial^{2} u}{\partial t^{2}}=(\lambda+2 \mu) \frac{\partial^{2} u}{\partial x^{2}}-\gamma \frac{\partial T}{\partial x}
$$

The constitutive equation will take the following form

$$
\sigma_{\mathrm{xx}}=(\lambda+2 \mu) \frac{\partial u}{\partial x}-\gamma T
$$

The Chandrasekharaiah and Tzou theory is such a modified of classical thermoelasticity model in which the Fourier law is replaced by an approximation of the equation

$$
q\left(x, t+\tau_{q}\right)=-K \nabla T\left(x, t+\tau_{\theta}\right)
$$

The model transmits thermoelastic disturbances in a wave-like manner if Equation $(*)$ is approximated by

$$
\left(1+\tau_{q} \frac{\partial}{\partial t}\right) q=-K\left(1+\tau_{\theta} \frac{\partial}{\partial t}\right) \nabla T,
$$

where $0 \leq \tau_{\theta}<\tau_{q}$.

Hence, we get the heat conduction equation in the context of dual-phase-lag model in the form

$$
K\left(1+\tau_{\theta} \frac{\partial}{\partial t}\right) \frac{\partial^{2} T}{\partial x^{2}}=\left(1+\tau_{q} \frac{\partial}{\partial t}\right)\left(\rho C_{E} \frac{\partial T}{\partial t}+\gamma T_{0} \frac{\partial^{2} u}{\partial x \partial t}\right)(3)
$$

where $\sigma_{x x}$ is the stress, $\lambda$ and $\mu$ are the Lamé constants, $\gamma$ is equal to $(3 \lambda+2 \mu) \alpha_{t}, \alpha_{t}$ is the thermal expansion coefficient, $K$ is the thermal conductivity, $C_{E}$ is the specific heat per unit mass at constant strain, $\rho$ is the density of the medium and $q_{i}$ is the heat flux vector.

Moreover, if we put $\tau_{\theta}=0$ and $\tau_{q}=\tau$ (the first relaxation time), fundamental equations possible for the Lord and Shulman's theory.

The initial and boundary conditions are taken as

$$
\begin{gathered}
\left.u(x, t)\right|_{t=0}=\left.\frac{\partial u(x, t)}{\partial t}\right|_{t=0}=0, x>0 \\
\left.T(x, t)\right|_{t=0}=\left.\frac{\partial T(x, t)}{\partial t}\right|_{t=0}=0, x>0, \\
\left.T(x, t)\right|_{x=0}=-T_{0} \mathrm{e}^{-t / \tau_{0}},\left.\frac{\partial u(x, t)}{\partial x}\right|_{x=0}=\left(1-\mathrm{e}^{-t / \tau_{0}}\right)
\end{gathered}
$$

where $\tau_{0}$ is constant and the regularity boundary conditions are $T(x, t), u(x, t)$ and $\sigma_{x x} \rightarrow 0$ as $x \rightarrow \infty$. 
For convenience, we shall use the following non-dimensional variables

$$
\begin{gathered}
x^{\prime}=c_{1} \eta x, \quad u^{\prime}=c_{1} \eta u, \quad t^{\prime}=c_{1}^{2} \eta t, \\
t_{q}{ }^{\prime}=c_{1}^{2} \eta \tau_{q}, \quad t_{\theta}{ }^{\prime}=c_{1}^{2} \eta \tau_{\theta}, \quad t_{0}{ }^{\prime}=c_{1}^{2} \eta \tau_{0}, \\
T^{\prime}=\frac{T}{T_{0}}, \quad \sigma_{x x}^{\prime}=\frac{\sigma_{x x}}{\mu},
\end{gathered}
$$

where

$$
\eta=\frac{\rho C_{E}}{K}, \quad c_{1}=\sqrt{\frac{\lambda+2 \mu}{\rho}}
$$

In terms of these variables, Equations (1)-(3) become (where the primes are suppressed for simplicity)

$$
\begin{gathered}
\frac{\partial^{2} u}{\partial t^{2}}=\frac{\partial^{2} u}{\partial x^{2}}-a \frac{\partial T}{\partial x} . \\
\left(1+t_{\theta} \frac{\partial}{\partial t}\right) \frac{\partial^{2} T}{\partial x^{2}}=\left(1+t_{q} \frac{\partial}{\partial t}\right)\left(\frac{\partial T}{\partial t}+g \frac{\partial^{2} u}{\partial x \partial t}\right) \\
\sigma_{\mathrm{xx}}=\beta^{2} \frac{\partial u}{\partial x}-b T
\end{gathered}
$$

where

$$
a=\frac{\gamma T_{0}}{\rho c_{1}^{2}}, \quad b=\frac{\gamma T_{0}}{\mu}, \beta^{2}=\frac{\lambda+2 \mu}{\mu}, g=\frac{\gamma}{\rho C_{E}} .
$$

and initial and boundary conditions will be

$$
\begin{gathered}
\left.u(x, t)\right|_{t=0}=\left.\frac{\partial u(x, t)}{\partial t}\right|_{t=0}=0, x>0, \\
\left.T(x, t)\right|_{t=0}=\left.\frac{\partial T(x, t)}{\partial t}\right|_{t=0}=0, x>0, \\
\left.T(x, t)\right|_{x=0}=-\mathrm{e}^{-t / t_{0}},\left.\frac{\partial u(x, t)}{\partial x}\right|_{x=0}=\left(1-\mathrm{e}^{-t / t_{0}}\right)
\end{gathered}
$$

\section{Solution in the Laplace Transform Domain}

We use the Laplace transform of both sides of the last equations which is defined in the form

$$
\bar{f}(s)=\int_{0}^{\infty} \mathrm{e}^{-s t} f(t) \mathrm{d} t .
$$

Hence, we obtain Equations (5-7) in the form

$$
\begin{gathered}
\left(\frac{\mathrm{d}^{2}}{\mathrm{~d} x^{2}}-s^{2}\right) \bar{u}=a \frac{\mathrm{d} \bar{T}}{\mathrm{~d} x}, \\
\left(\left(1+t_{\theta} s\right) \frac{\mathrm{d}^{2}}{\mathrm{~d} x^{2}}-s\left(1+t_{q} s\right)\right) \bar{T}=s\left(1+t_{q} s\right) g \frac{\mathrm{d} \overline{\mathrm{u}}}{\mathrm{d} x}
\end{gathered}
$$

$$
\bar{\sigma}_{\mathrm{xx}}=\beta^{2} \frac{\mathrm{d} \bar{u}}{\mathrm{~d} x}-b \bar{T}
$$

where the over bar symbol denotes its Laplace transform and s denotes the Laplace transform parameter.

The boundary conditions (8) in the Laplace transform can be expressed in the form

$$
\begin{aligned}
& \left.\bar{T}(x, t)\right|_{x=0}=-\frac{t_{0}}{1+s t_{0}} \\
& \left.\frac{\mathrm{d} \bar{u}(x, t)}{\mathrm{d} x}\right|_{x=0}=\frac{t_{0}}{s\left(1+s t_{0}\right)}
\end{aligned}
$$

Introducing the thermoelastic potential function $\phi$ defined by the relation

$$
\bar{u}=\frac{\mathrm{d} \bar{\phi}}{\mathrm{d} x}
$$

Equations (9-10) reduce to

$$
\begin{gathered}
\left(\frac{\mathrm{d}^{2}}{\mathrm{~d} x^{2}}-s^{2}\right) \bar{\phi}=a \bar{T}, \\
\left(\left(1+t_{\theta} s\right) \frac{\mathrm{d}^{2}}{\mathrm{~d} x^{2}}-s\left(1+t_{q} s\right)\right) \bar{T}=s\left(1+t_{q} s\right) g \frac{\mathrm{d}^{2} \bar{\phi}}{\mathrm{d} x^{2}} .
\end{gathered}
$$

Eliminating $T$ from Equations (14) and (15), we obtain

$$
\left(\frac{\mathrm{d}^{4}}{\mathrm{~d} x^{4}}-\left[s^{2}+P(1+\varepsilon)\right] \frac{\mathrm{d}^{2}}{\mathrm{~d} x^{2}}+s^{2} P\right) \bar{\phi}=0,
$$

where $\varepsilon$ is the mechanical coupling constant defined by $\varepsilon=a g$ and $P=\frac{s\left(1+t_{q} s\right)}{\left(1+t_{\theta} s\right)}$.

The solutions of Equation (16) bounded at infinity can be written in the form:

$$
\bar{\phi}=A_{1} \mathrm{e}^{-m_{1}}+A_{2} \mathrm{e}^{-m_{2}}
$$

where $A_{1}$ and $A_{2}$ are parameters depending on $s$ to be determined from the boundary conditions, $m_{1}$ and $m_{2}$ are the roots with positive real parts of the characteristic equation

$$
m^{4}-\left[s^{2}+P(1+\varepsilon)\right] m^{2}+s^{2} P=0
$$

$m_{1}$ and $m_{2}$ are given by

$$
m_{1,2}=\sqrt{\frac{\left[s^{2}+P(1+\varepsilon)\right]+\sqrt{\left[s^{2}+P(1+\varepsilon)\right]^{2}-4 s^{2} P}}{2}}
$$

The expression for displacement and temperature can be written in the forms

$$
\bar{u}=-m_{1} A_{1} \mathrm{e}^{-m_{1}}-m_{2} A_{2} \mathrm{e}^{-m_{2}}
$$




$$
\bar{T}=\frac{\left(m_{1}^{2}-s^{2}\right)}{a} A_{1} \mathrm{e}^{-m_{1}}+\frac{\left(m_{2}^{2}-s^{2}\right)}{a} A_{2} \mathrm{e}^{-m_{2}}
$$

Substituting from Equations (20) and (21) into Equation (11), we obtain

$$
\begin{aligned}
& \bar{\sigma}_{x x}=\left(m_{1}^{2}\left(\beta^{2}-\frac{b}{a}\right)+\frac{b s^{2}}{a}\right) A_{1} \mathrm{e}^{-m_{1}} \\
& +\left(m_{2}^{2}\left(\beta^{2}-\frac{b}{a}\right)+\frac{b s^{2}}{a}\right) A_{2} \mathrm{e}^{-m_{2}}
\end{aligned}
$$

From the boundary conditions (12), it follows that

$$
\begin{gathered}
A_{1}=-\frac{m_{2}^{2}\left(1+s t_{0}\right)-s^{2}}{s^{3}\left(1+s t_{0}\right)\left(m_{1}^{2}-m_{2}^{2}\right)}, \\
A_{2}=\frac{m_{1}^{2}\left(1+s t_{0}\right)-s^{2}}{s^{3}\left(1+s t_{0}\right)\left(m_{1}^{2}-m_{2}^{2}\right)}
\end{gathered}
$$

\section{Numerical Inversion of the Laplace Transform}

In order to determine the conductive and thermal temperature, displacement and stress distributions in the time domain, we adopt a numerical inversion method based on a Fourier series expansion [15]. In this method, the inverse $\mathrm{g}(\mathrm{t})$ of the Laplace transform $\mathrm{g}(\mathrm{s})$ is approximated by the relation

$$
g(t)=\frac{e^{c t}}{t_{1}}\left(\frac{\bar{g}(c)}{2}+\operatorname{Re} \sum_{k=1}^{N} e^{i k t \pi / t_{1}} \bar{g}\left(c+\frac{i k \pi}{t_{1}}\right)\right), 0 \leq t \leq t_{1},
$$

where $\operatorname{Re}$ is the real part and $i=\sqrt{-1}$ is imaginary number unit and $N$ is a sufficiently large integer representing the number of terms in the truncated infinite Fourier series. For faster convergence, numerous numerical experiments have shown that the value of $c$ satisfies the relation $c t \approx 4.7 \quad$ Tzou [9].

$N$ must chosen such that

$$
\mathrm{e}^{c t} \operatorname{Re}\left[\mathrm{e}^{i k t \pi / t_{1}} \bar{g}\left(c+\frac{i k \pi}{t_{1}}\right)\right] \leq \varepsilon_{1}
$$

where $\varepsilon_{1}$ is a persecuted small positive number that corresponds to the degree of accuracy to be achieved. The parameter $c$ is a positive free parameter that must be greater than the real parts of all singularities of $\bar{g}(s)$. The optimal choice of $\mathrm{c}$ was obtained according to the criteria described in [15].

Formula (23) was used to invert the Laplace transforms in Equations (20)-(22), given the temperature, stress, and displacement distributions.

\section{Numerical Results}

Now, we will consider a numerical example for which computational results are given. For this purpose, Copper is taken as the thermoelastic material for which we take the following values of the different physical constants [16]

$$
\begin{gathered}
K=368, \quad \alpha_{t}=1.78 \times 10^{-5}, C_{E}=383.1, \quad g=1.61, \\
\rho=8954, \lambda=7.76 \times 10^{10}, \mu=3.86 \times 10^{10}, \\
\mu=8886.73, \quad \beta^{2}=4, \mathrm{~T}_{0}=293, \varepsilon=0.0168 .
\end{gathered}
$$

The non-dimensional temperature $T$, displacement $u$, and stress component $\sigma_{x x}$ distributions were evaluated on the $x$-axis. Further by setting the phase-lag of the heat flux $t_{\theta}$ to zero, the results due to the Lord and Shulman's theory are obtained. The computations were carried out for one value of time, namely for $t=0.1$.

The graphs of the temperature, displacement and thermal stress due to phase-lag of the heat flux $t_{q}$ are exhibited graphically in Figures 1-3. The results carried

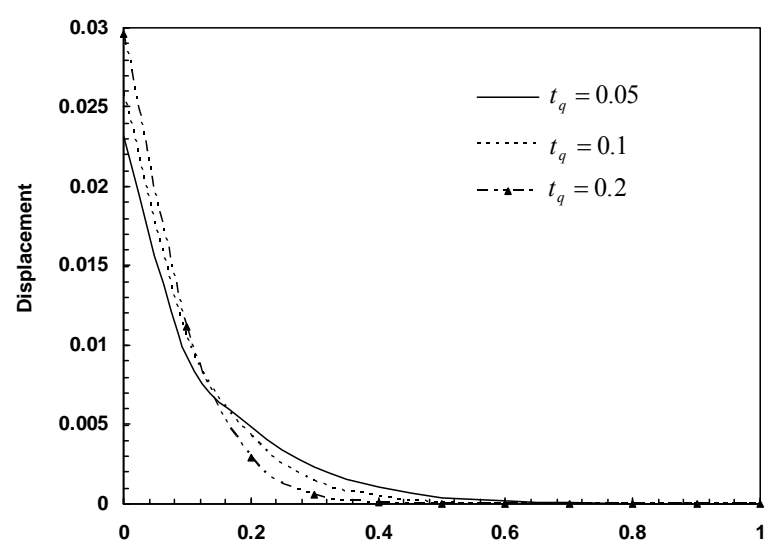

Figure 1. Displacement vs. distance for different values of phaselag of the heatflux.

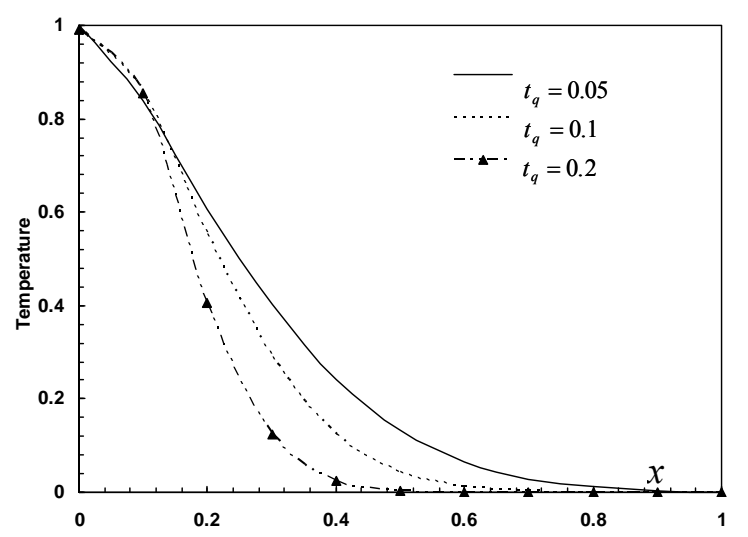

Figure 2. Temperature vs. distance for different values of phaselag of heatflux. 


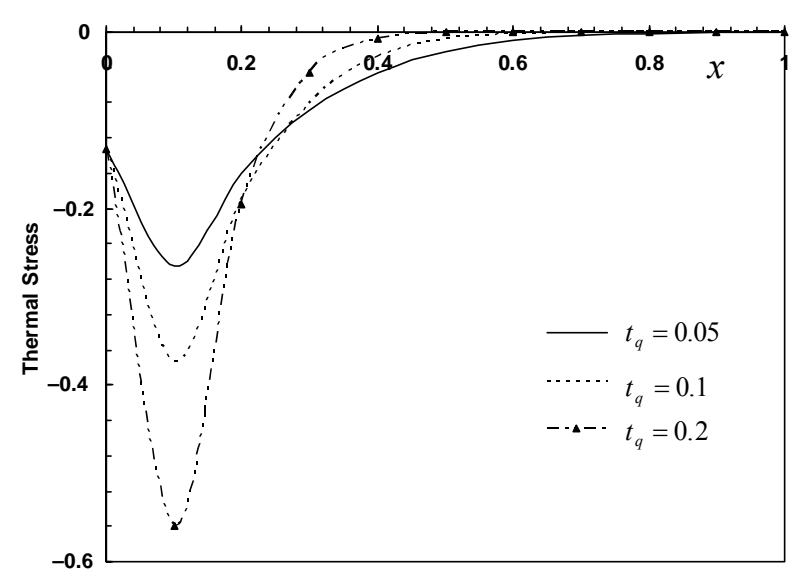

Figure 3. Thermal stress vs. distance for different values of phaselag of heatflux.

out for three values of $t_{q}$, namely $t_{q}=0.05,0.1$ and 0.2 . We can deduce that:

1) The parameter $t_{q}$ has significant effects on all the fields.

2) The wave has a finite speed of propagation. This result shows that the DPL model agree with the generalized thermoelasticity.

3) The temperature and displacement starts with its maximum value at the origin and decreases until attaining zero beyond a wave front for the generalized theory, which agree with the boundary conditions.

4) The magnitude of the stress increases rapidly as $x$ increases and it attains a peak value at $x=0.1$, thereafter it decreases slowly with increasing $x$ whereas the magnitude of the peak value is reduced with the increase of $t_{q}$.

Figures 4-6 show the heat, the displacement, and the stress respectively with distance $x$ at the same instance $t=0.1$ with different values of the phase-lag of temperature gradient parameter $t_{\theta}\left(t_{\theta}=0=t_{q}\right)$ which means coupled thermoelasticity model of Biot, $t_{\theta}=0$ which means generalized thermoelasticity model of Lord and Shulman and for $t_{\theta} \neq 0 \quad\left(t_{\theta}=0.05\right.$ and $\left.t_{\theta}=0.08\right)$ means that DPL model and we found that, the parameter $t_{\theta}$ has a significant effects on all the fields.

In all these figures, it is clear that the values of solutions for L-S theory are large in comparison with those for DPL model. This may be due to the nature of the boundary conditions, which we take.

\section{Conclusions}

In the framework of this article, a problem of a halfspace whose surface is rigidly fixed and subjected to the effects of a thermal shock on the surface within the context of the theory of generalized thermoelasticity pro-

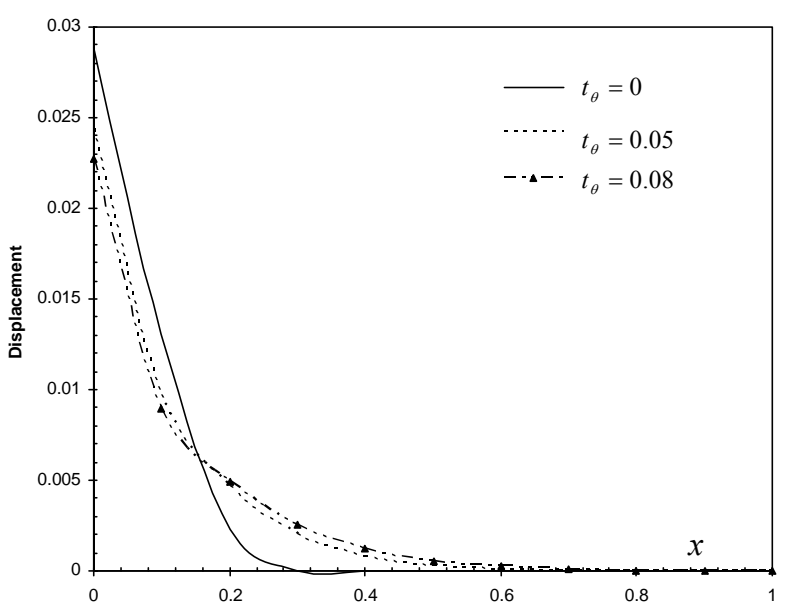

Figure 4. Displacement vs. distance for different values of phaselag of gradient of temperature.

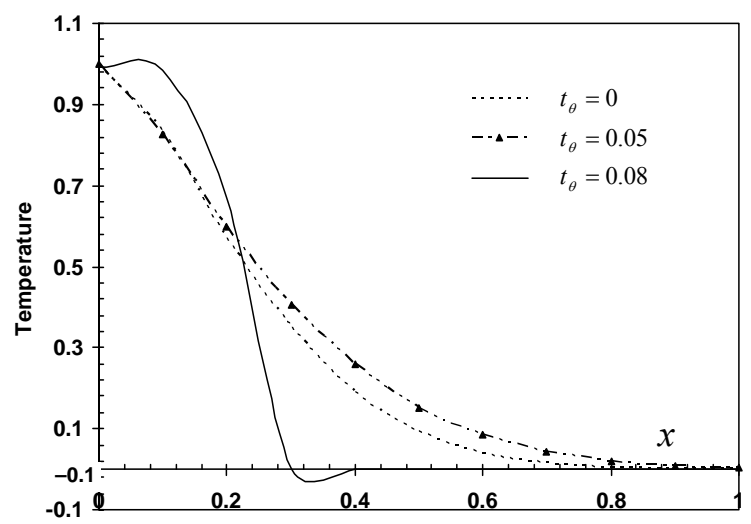

Figure 5. Temperature vs. distance for different values of phaselag of gradient of temperature.

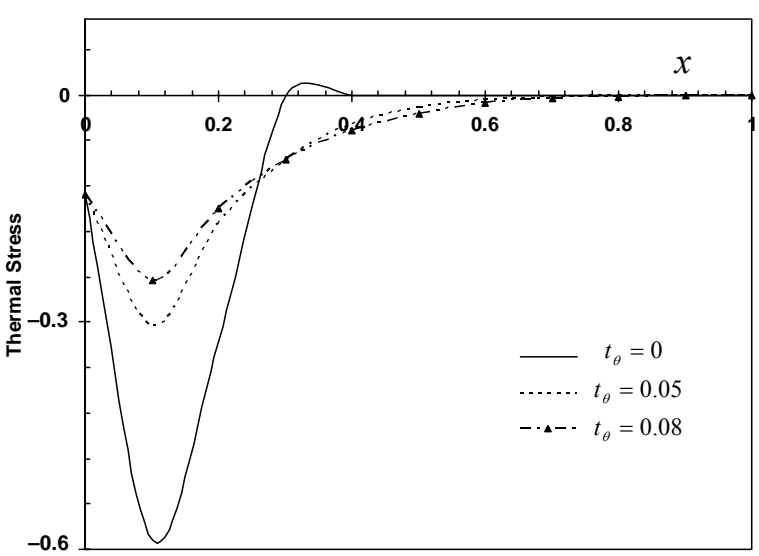

Figure 6. Thermal stress vs. distance for different values of phaselag of gradient of temperature.

posed by Tzou.

According to the above results, we can conclude that:

1) As the phase-lag of the heat flux $t_{q}$ constant increases the corresponding components of temperature, 
displacement and stress decrease.

2) The increases of the phase-lag of gradient of temperature $t_{\theta}$ decrease the components of temperature, displacement and stress distributions.

3) We found that, the parameters $t_{q}$ and $t_{\theta}$ have significant effects on all the fields.

4) The phenomenon of finite speeds of propagation is manifested in all these figures.

The comparison of different theories of thermoelasticity, i.e. Lord and Shulman theory and Chandrasekharaiah and Tzou model is carried out.

\section{References}

[1] M. Biot, "Thermoelasticity and Irreversible ThermodyNamics," Journal of Applied Physics, Vol. 27, No. 3, 1956, pp. 240-253. doi:10.1063/1.1722351

[2] H. Lord and Y. Shulman, "A Generalized Dynamical Theory of Thermoelasticity," Journal of the Mechanics and Physics of Solids, Vol. 15, No. 5, 1967, pp. 299-309. doi:10.1016/0022-5096(67)90024-5

[3] R. Dhaliwal and H. Sherief, "Generalized Thermoelasticity for Anisotropic Media," Quarterly of Applied Mathematics, Vol. 33, 1980, pp. 1-8.

[4] A. E. Green and N. Laws, "On the Entropy Production Inequality," Archive for Rational Mechanics and Analysis, Vol. 45, No. 1, 1972, pp. 47-53. doi:10.1007/BF00253395

[5] A. E. Green and K. A. Lindsay, "Thermoelasticity," Journal of Elasticity, Vol. 2, No. 1, 1972, pp. 1-7. doi:10.1007/BF00045689

[6] A. E. Green and P. M. Naghdi, "Thermoelasticity Without Energy Dissipation," Journal of Elasticity, Vol. 31, No. 3, 1993, pp. 189-208. doi:10.1007/BF00044969

[7] D. Y. Tzou, "Macro- to Microscale Heat Transfer: The Lagging Behavior," 1st Edition, Taylor \& Francis, Washington, 1996.

[8] D. Y. Tzou, "A Unified Approach for Heat Conduction
From Macro- to Micro- Scales," Journal of Heat Transfer, Vol. 117, No. 1, 1995, pp. 8-16. doi:10.1115/1.2822329

[9] D. Y. Tzou, "Experimental Support for the Lagging Behavior in Heat Propagation," Journal of Thermophysics and Heat Transfer, Vol. 9, 1995, pp. 686-693. doi: $10.2514 / 3.725$

[10] V. Danilovskaya, "Thermal Stresses in an Elastic Halfspace Due to Sudden Heating of Its Boundary," Prikl Mat. Mekh., In Russian, Vol. 14, 1950, pp. 316-324.

[11] D. S. Chandrasekharaiah and K. S. Srinath, "One-Dimensional Waves in a Thermoelastic Half-Space Without Energy Dissipation," International Journal of Engineering Science, Vol. 34, No. 13, 1996, pp. 1447-1455. doi:10.1016/0020-7225(96)00034-1

[12] S. K. Roychoudhuri and P. S. Dutta, "Thermoelastic Interaction Without Energy Dissipation in an Infinite Solid with Distributed Periodically Varying Heat Sources," International Journal of Solids Structures, Vol. 42, 2005, pp. 4192-4203.

[13] H. Sherief, and R. Dhaliwal, "Generalized One-Dimensional Thermal Shock Problem for Small Times," Journal of Thermal Stresses, Vol. 4, No. 3-4, 1981, pp. 407-420. doi: 10.1080/01495738108909976

[14] M. N. Allam, K. A. Elsibai and A. E. Abouelregal, "Magneto-Thermoelasticity for an Infinite Body with a Spherical Cavity and Variable Material Properties Without Energy Dissipation," International Journal of Solids and Structures, Vol. 47, No. 20, 2010, pp. 2631-2638. doi:10.1016/j.ijsolstr.2010.04.021

[15] G. Honig and U. Hirdes, "A Method for the Numerical Inversion of the Laplace Transform," Journal of Computational and Applied Mathematics, Vol. 10, No. 1, 1984, pp. 113-132. doi:10.1016/0377-0427(84)90075-X

[16] H. Youssef, "Thermomechanical Shock Problem of Generalized Thermoelastic Infinite Body with a Cylindrical Cavity and Material Properties Depends on the Reference Temperature," Journal of Thermal Stresses, Vol. 28, No. 5, 2005, pp. 521-532. doi:10.1080/01495730590925029 